\title{
Determination of interfacial properties between metal film and ceramic substrate with an adhesive layer
}

\author{
H.F. Zhao ${ }^{\mathrm{a}, \mathrm{b}, *}$, M. Chen ${ }^{\mathrm{a}}$, Y. Jin ${ }^{\mathrm{a}}$ \\ a MOE-Key Laboratory of Petroleum Engineering, China University of Petroleum, Beijing 102249, PR China \\ ${ }^{\mathrm{b}}$ State-Key Laboratory of Nonlinear Mechanics (LNM), Institute of Mechanics, Chinese Academy of Science, Beijing 100080, PR China
}

\section{A R T I C L E I N F O}

\section{Article history:}

Received 28 July 2007

Accepted 16 April 2008

Available online 25 April 2008

\section{Keywords:}

B. Film and sheet

D. Bonding

G. Destructive testing

\begin{abstract}
A B S T R A C T
Peel test measurements and inverse analysis to determine the interfacial mechanical parameters for the metal film/ceramic system are performed, considering that there exist an epoxy interface layer between film and ceramic. In the present investigation, $\mathrm{Al}$ films with a series of thicknesses between 20 and $250 \mu \mathrm{m}$ and three peel angles of 90,135 and $180^{\circ}$ are considered. A finite element model with the cohesive zone elements is used to simulate the peel test process. The finite element results are taken as the training data of a neural network in the inverse analysis. The interfacial cohesive energy and the separation strength can be determined based on the inverse analysis and peel experimental result.
\end{abstract}

(c) 2008 Elsevier Ltd. All rights reserved.

\section{Introduction}

Thin film/substrate systems have been widely used in engineering and the research on strength, ductility and reliability of film/ substrate systems has attracted much interest in recent years. Thin film delamination is a major failure formation in these systems. The delaminating process can be characterized by a two-parameter criterion. These two parameters are the interfacial fracture toughness $\Gamma_{0}$ and the adhesion strength $\hat{\sigma}[1-5]$. Usually, interfacial fracture toughness (or called cohesive energy) undergoes the great attention in the elastic case or small-scale yielding case of the adherends. When plastic dissipation cannot be neglected, one needs to consider another parameter effect additionally [6,7]. Fig. 1 shows a sketch of the peel test with the film thickness $t$, peel force $P$ and peel angle $\Phi$. The right hand side part of Fig. 1 shows the cohesive zone (CZ) model by which the definition of the interface parameters is given [6-10]. There are two important parameters in the CZ model, $\left(\Gamma_{0}, \hat{\sigma}\right)$. The determination of $\left(\Gamma_{0}, \hat{\sigma}\right)$ for a film/substrate system is the most important goal in the peel test. Through the peel test measurements one can record both the peel force $P$ and the deformation information of the film. From energy balance at the steady-state peeling, one can obtain a relationship between energy release rate $P(1-\cos \Phi)$ with interfacial fracture toughness $\Gamma_{0}$ as well as plastic dissipation energy $\Gamma_{\mathrm{P}}$ :

$P(1-\cos \Phi)=\Gamma_{0}+\Gamma_{\mathrm{P}}$

\footnotetext{
* Corresponding author. Address: MOE-Key Laboratory of Petroleum Engineering, China University of Petroleum, Beijing 102249, PR China.

E-mail address: zhaohf@vip.163.com (H.F. Zhao).
}

In most metal film cases $\Gamma_{\mathrm{P}}$ is a major contribution to the energy release rate $P(1-\cos \Phi)$. So an appropriate method is needed to determine $\Gamma_{0}$, when film deforms plastically [3-5,8-13].

In order to determine $\Gamma_{0}$ using the peel test, in the previous methods, a beam bending model was adopted $[4,5]$. However, this model is suitable for the cases of the thick film and weak interface adhesion [14-16].

In this paper we will focus our attention on the determination of interfacial parameters for thin $\mathrm{Al}$ films with thickness ranging between 20 and $250 \mu \mathrm{m}$ bonded to a ceramic substrate $\left(\mathrm{Al}_{2} \mathrm{O}_{3}\right)$ with a type of epoxy adhesive. Peel test measurements are performed and a general inverse analysis method based on neural network to determine the interfacial mechanical parameters is presented. Three cases of peel angles 90,135 and $180^{\circ}$ are considered. A plane strain FE model with the cohesive zone elements is adopted to simulate the peel test process. The simulation results are used as the training data to train a neural network. The trained network is adopted to predict the interfacial cohesive energy $\Gamma_{0}$ and separation strength $\hat{\sigma}$.

\section{Experimental method}

2.1. Overview

Peel tests are performed for the Al films with a series of thicknesses, 20, 50, 80, $100,200,225$ and $250 \mu \mathrm{m}$, bonded to $4.5 \mathrm{~mm}$ thick $\mathrm{Al}_{2} \mathrm{O}_{3}$ substrates with one type of epoxy/polyimide paste adhesive. The mass ratio of epoxy to polyimide in the adhesive is 1.5 . The adhesive shows ductile property in the peel tests, so it is called ductile adhesive in the following.

It is crucial to control the adhesive layer thickness $d$ in preparing the samples. In our peel tests the adhesive layer thickness is kept constant by adding some small $\mathrm{SiO}_{2}$ spheres to the adhesive, see Fig. 2. The adhesive layer thickness is kept at $20 \mu \mathrm{m}$ in this paper. 


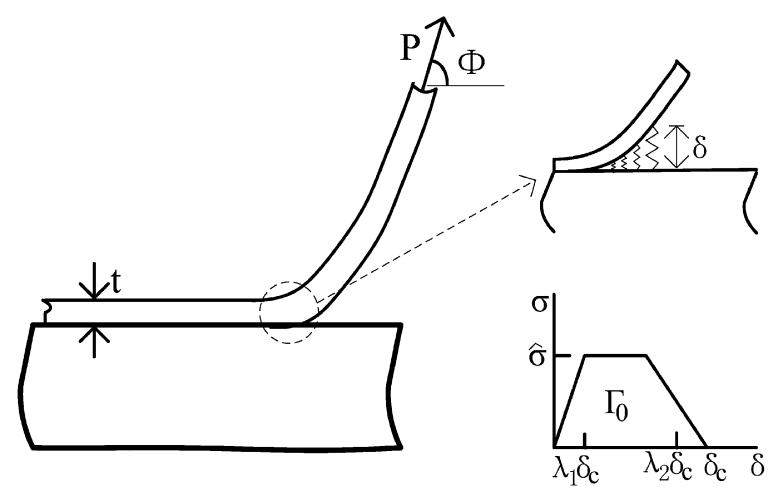

Fig. 1. Peel test configuration and sketch of the cohesive zone model.

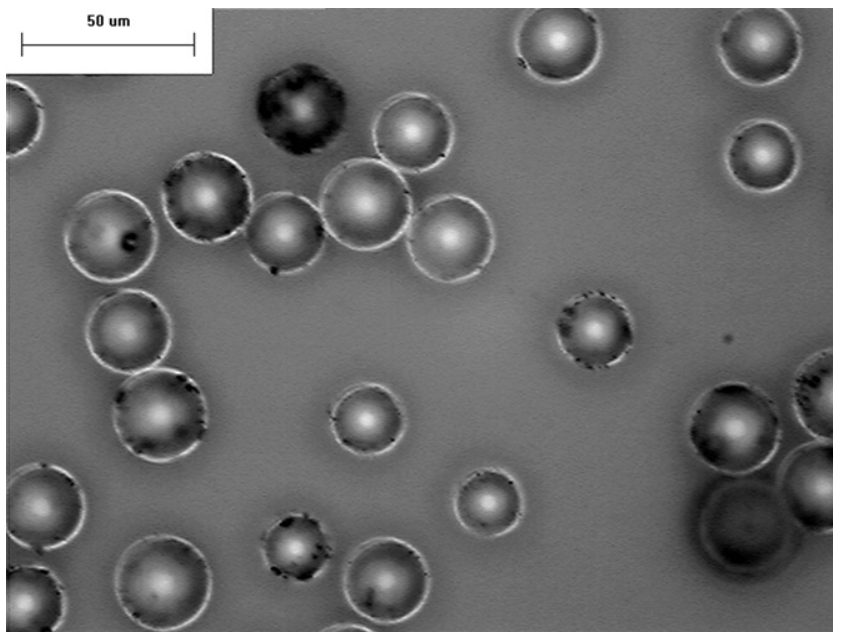

Fig. 2. $\mathrm{SiO}_{2}$ spheres used to control the adhesive layer thickness.

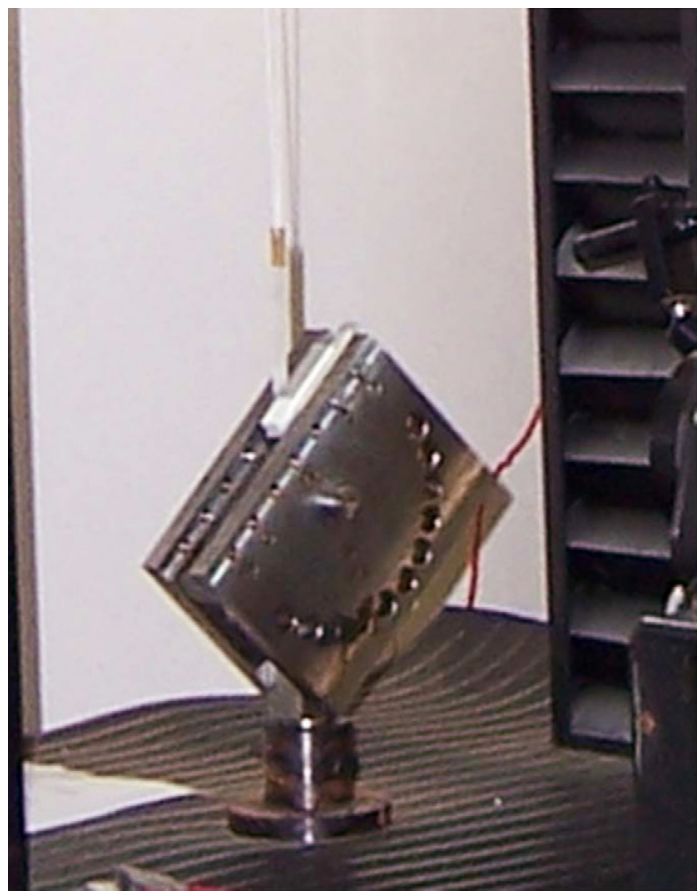

Fig. 3. Peel test rig made specifically for the current research.
All the peel tests are performed using a standard tensile testing machine with a small-scale peel test rig specifically designed for the current research (see Fig. 3). Several peel angles can be easily maintained with this peel test rig. A Questar microscope with long focus is used to observe the crack growth and take micrographs. The thin films are difficult to be fixed directly to the testing machine. So in order to protect the films from tearing, piece of adhesive tape is used to connect the film to some small metal sheet, and a thin nylon thread is used to connect the metal sheet to the testing machine. Since the nylon thread is about one meter long and the crosshead displacement never exceeds $30 \mathrm{~mm}$, the change of the peel angle during peel test is smaller than $\operatorname{arctg}(0.03) \approx 1.5^{\circ}$. Therefore, the peel angle is kept approximately during peel process.

Peel velocity $v_{\text {crack }}$ is kept constant $(1 \mathrm{~mm} / \mathrm{min}$ ) during peel process, i.e.:

$v /(1-\cos \Phi)=v_{\text {crack }}=$ const

where $v$ is the moving velocity of the crosshead and $\Phi$ is the peel angle.

Table 1

Material parameters of the Al films

\begin{tabular}{lllll}
\hline $\begin{array}{l}\text { Film thickness } \\
(\mu \mathrm{m})\end{array}$ & $\begin{array}{l}\text { Young's modulus }^{\mathrm{a}} \\
(\mathrm{GPa})\end{array}$ & $\begin{array}{l}\text { Poisson's }_{\text {ratio }^{\mathrm{a}}} \\
20\end{array} \mathrm{l}^{\text {Yield strength }}(\mathrm{MPa})$ & $\begin{array}{l}\text { Strain hardening } \\
\text { exponent }\end{array}$ \\
\hline 50 & 71 & 0.31 & 36.3 & 0.238 \\
80 & 71 & 0.31 & 34.0 & 0.243 \\
100 & 71 & 0.31 & 33.2 & 0.246 \\
200 & 71 & 0.31 & 32.8 & 0.249 \\
225 & 71 & 0.31 & 32.0 & 0.251 \\
250 & 71 & 0.31 & 31.9 & 0.250 \\
& 71 & 0.31 & 31.8 & 0.250 \\
\hline
\end{tabular}

a From materials handbook.
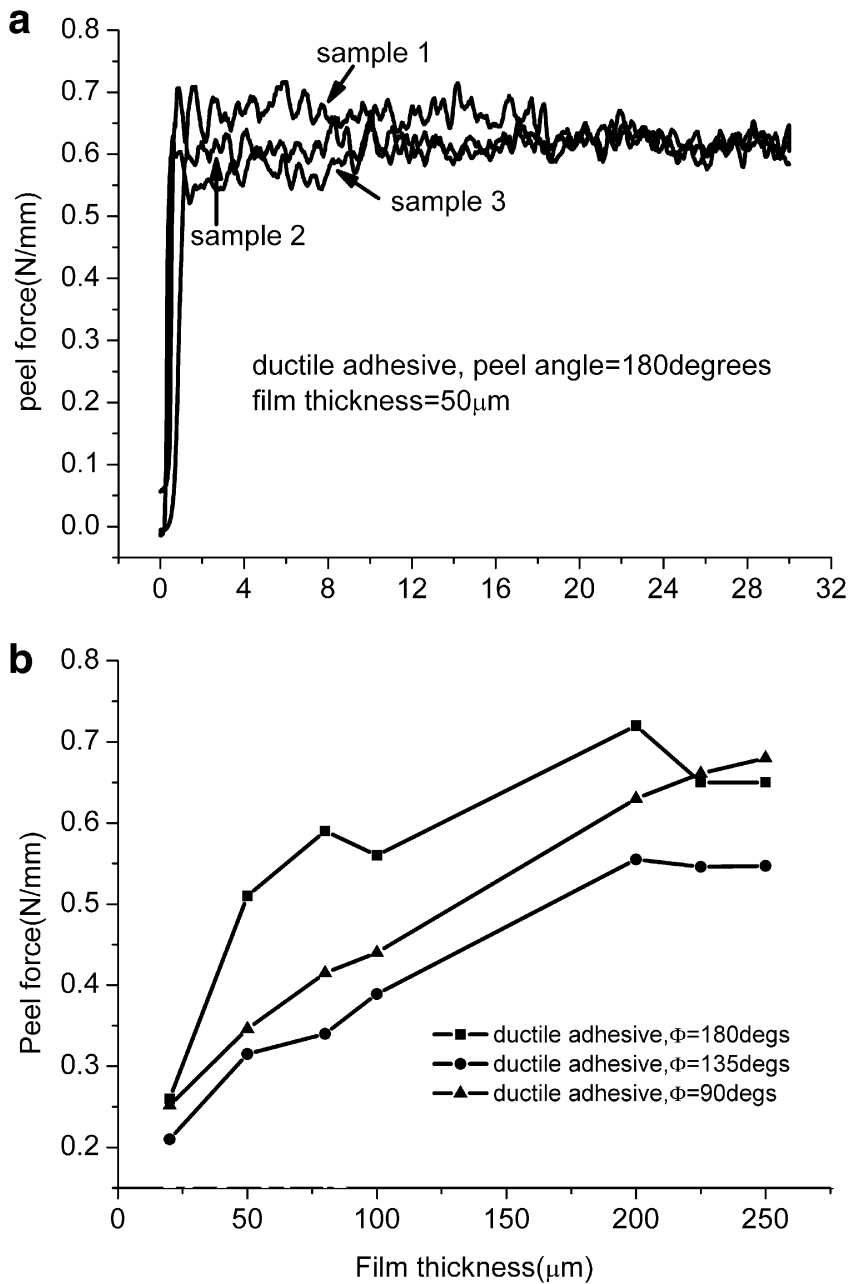

Fig. 4. (a) Variations of the peel force vs. crosshead displacement, and (b) variations of the steady-state peel force vs. film thickness. 


\subsection{Experimental results}

\subsubsection{Materials}

2.2.2.1. Film. The Al film is tested using the uniaxial tension and the stress-strain curve is fitted using the following piece power-law hardening relations:

$\sigma=\left\{\begin{array}{cl}E \varepsilon, & \left(\sigma \leqslant \sigma_{y}\right) \\ \frac{\sigma_{y}}{\left(\sigma_{\mathrm{y}} / E\right)^{n}} \varepsilon^{n}, & \left(\sigma \geqslant \sigma_{y}\right)\end{array}\right.$

where $n$ is strain hardening exponent. Table 1 shows the fitting material parameters for the Al films.

2.2.2.2. Material $\mathrm{Al}_{2} \mathrm{O}_{3}$. Substrate material, $\mathrm{Al}_{2} \mathrm{O}_{3}$ is treated as an elastic material with Young's modulus $E=350 \mathrm{GPa}$ and poisson's ration $v=0.3$ in the present research.

\subsubsection{Peel test results}

The curves of peel force vs. crosshead displacement are recorded during the peel tests. Fig. 4a shows some typical curves of peel force vs. crosshead displacement. From Fig. 4a, obviously the peel process mainly consists of two stages: initial peeling and steady-state peeling. In the present research, we pay attention to the steady-state peeling.
At least three samples are used to do peel tests for each film thickness and each peel angle. The mean value of the measured steady-state peel forces is taken as a function of the film thickness. The functions are plotted in Fig. $4 \mathrm{~b}$. The steady-state peel force increases with increasing film thickness until reaches at the stable value when film thickness is larger than $200 \mu \mathrm{m}$. It should be noted that peel forces for $90^{\circ}$ is larger than $135^{\circ}$, but lower than $180^{\circ}$ for a given film thickness. This result may be explained by Eq. (1):

$P=\frac{\Gamma_{0}+\Gamma_{p}}{1-\cos \Phi}$

From 90 to $180^{\circ}, \Gamma_{\mathrm{p}}$ increases because the curvature radius of the film at the crack tip decreases which means the film is "bend more". On the other hand, $1-\cos \Phi$ also increases from 1 to 2 when peel angle increases from 90 to $180^{\circ}$. The two factors determine that peel force will reach minimum at some peel angle between 90 and $180^{\circ}$.

Two typical configurations of the peeled films near the crack tip are shown in Fig. 5a and b for peel angle $\Phi=180^{\circ}$ and $135^{\circ}$, respectively. All peeled films are debonded along the interface between the film and the adhesive layer.

For each peel test with $\Phi=180^{\circ}$, the curvature radius of the film at the crack tip is also measured by using multiple points to fit the configuration of the film at the crack tip on the micrograph taken by the Questar measuring system, see Fig. 5a. The a

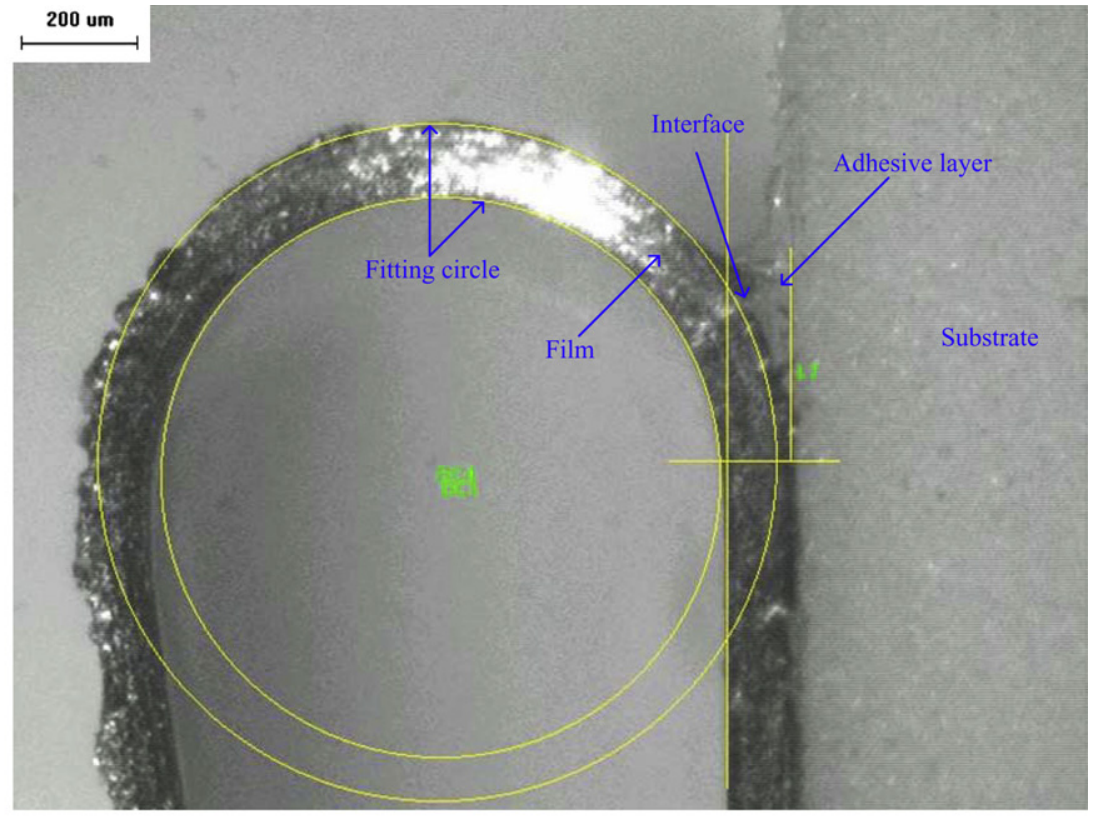

b

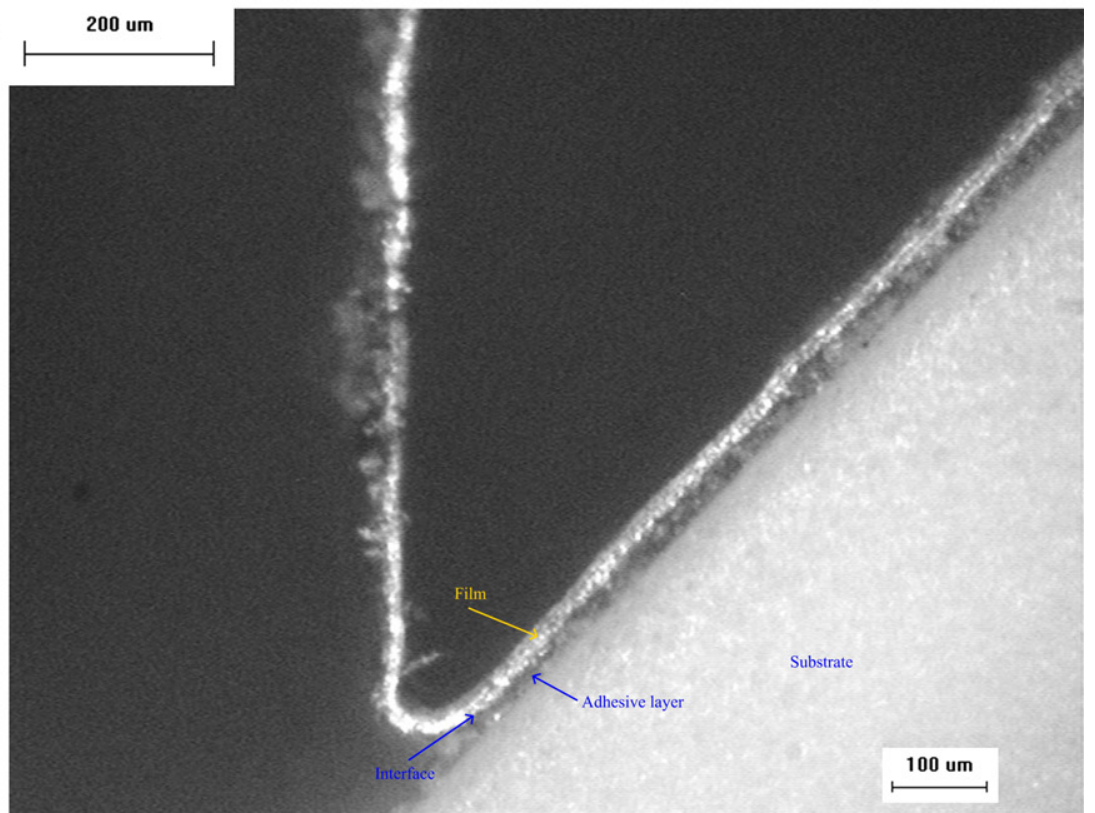

Fig. 5. (a) Peel angle $180^{\circ}$, film thickness $100 \mu \mathrm{m}$; and (b) peel angle $135^{\circ}$, film thickness $20 \mu \mathrm{m}$. 


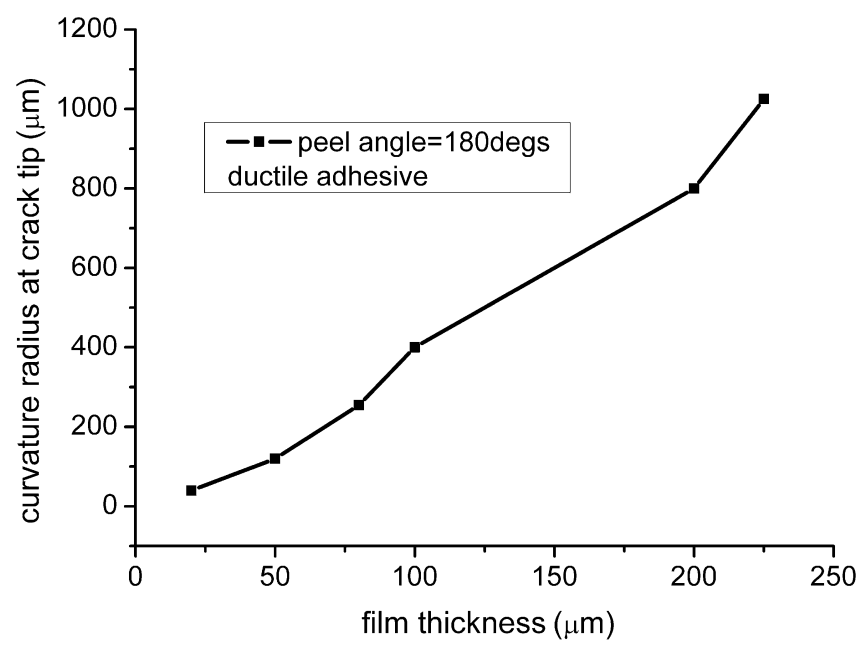

Fig. 6. The curvature radius of the film at the crack tip.

measured result is shown in Fig. 6 . It should be noted the curvature radius of the film varies in the crack tip region, and the result in Fig. 6 is the minimum value which is the true curvature radius at the crack tip.

\section{Theoretical method: FE simulations and neural network inverse analysis}

\subsection{FE model with the $C Z$ model}

Since in the peel test the film width $(10 \mathrm{~mm})$ is much larger than its thickness $(20-250 \mu \mathrm{m})$, the peel test problem can be treated as the plane strain problem. The FE simulation using ABAQUS version 6.5 is performed. Eq. (3) is used to characterize the stress strain relation of the Al film. Large deformation, von Mises yield criterion and isotropic strain hardening will be considered in our FE model. Moreover, for substrate material, since the $\mathrm{Al}_{2} \mathrm{O}_{3}$ substrate undergoes the very small deformation during the peel tests, it can treated as an elastic material with Young's modulus $E=350 \mathrm{GPa}$ and Poisson's ration $v=0.3$.

A single layer of $C Z$ elements [6-10] is employed to represent the adhesive layer. The interface parameters governing the traction separation law are the interface fracture toughness $\Gamma_{0}$, separation strength $\hat{\sigma}$ and the factors $\lambda_{1}$ and $\lambda_{2}$, as described in Fig. 1. Earlier studies show that the shape of the traction separation law is relatively unimportant, and two most important parameters are $\Gamma_{0}$ and $\hat{\sigma}$ [17]. In our FE model take $\lambda_{1}=0.15, \lambda_{2}=0.5$. For the convenience of exerting load on the film to simulate peel test, a rigid body is settled at the free end of the film. At first the free end of the film is rotated by the peel angle and then the film is peeled along this direction. The film and the substrate are meshed using bi-linear rectangular elements with four nodes and four integration points. The film undergoes large bending deformation during the peeling, so at least four layer elements should be divided along the thickness of the film to capture large deformation information. Since Young's modulus of the substrate $\mathrm{Al}_{2} \mathrm{O}_{3}$ is about five times that of the $\mathrm{Al} \mathrm{film}$ and the substrate undergoes small deformation during the peeling, sparse mesh is adopted within it. Fig. 7 shows a typical mesh used in our FE simulations.

\subsection{Inverse analysis using neural network to predict $\Gamma_{0}$ and $\hat{\sigma}$}

Since both the interfacial fracture energy $\Gamma_{0}$ and separation strength $\hat{\sigma}$ are most important parameters in the interface fracture research [5], we select them as the target to be measured in the present research. Here, an inverse analysis method is presented

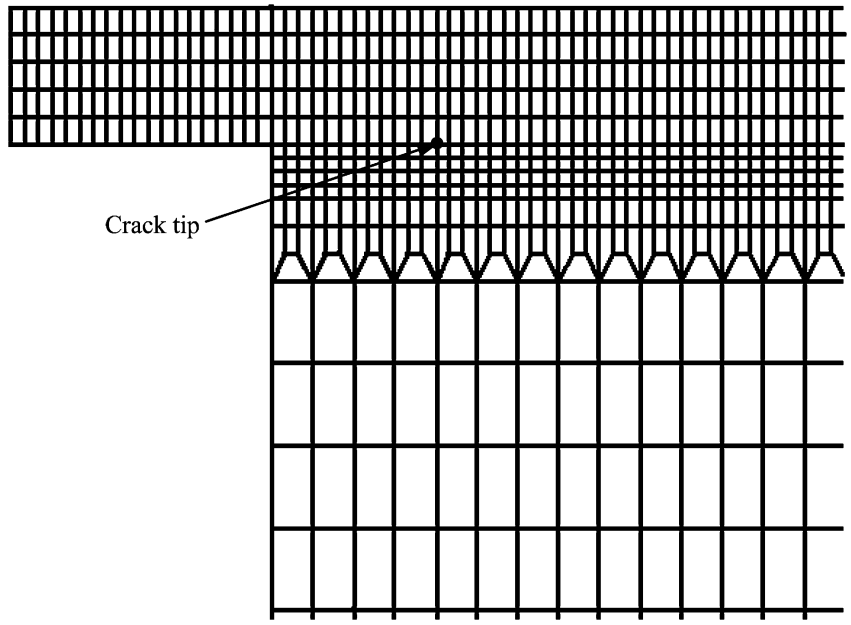

Fig. 7. A typical mesh used in the FE calculations.

to identify the parameters $\Gamma_{0}$ and $\hat{\sigma}$ by using the artificial neural network method.

For the film thickness $50 \mu \mathrm{m}$ and peel angle $180^{\circ}$, both the peel force $P$ and the bending curvature radius $r$ of the film at the crack tip can be described uniquely by interfacial parameters $\Gamma_{0}$ and $\hat{\sigma}$ :

$P=f_{1}\left(\Gamma_{0}, \hat{\sigma}\right)$

$r=g_{1}\left(\Gamma_{0}, \hat{\sigma}\right)$

We also have the inverse relations:

$\Gamma_{0}=f_{2}(P, r)$

$\hat{\sigma}=g_{2}(P, r)$

Both $f_{2}$ and $g_{2}$ can be determined numerically by using the neural network method.

In the inverse analysis based on the neural network method, the finite element solutions are used first as training data to train the neural network. Given a series of values $\left(\Gamma_{0}^{i}, \hat{\sigma}^{i}\right)$, one can obtain the same number of values $\left(P^{i}, r^{i}\right)$ by using the finite element method. The obtained results are used as input data for training the neural network, while values $\left(\Gamma_{0}^{i}, \hat{\sigma}^{i}\right)$ are used as target data. From the experimental results shown in Fig. $4 \mathrm{~b}$, one can find the region of interfacial fracture energy $\Gamma_{0}<0.2 \mathrm{~N} / \mathrm{mm}$. So for the series $\left(\Gamma_{0}^{i}, \hat{\sigma}^{i}\right)$, we take 10 values of $\Gamma_{0}$ in the range $(0.02,0.2)$ and ten values of $\hat{\sigma}$ in a large range $(5,50)$. Through finite element calculation, we have 100 values of $\left(P^{i}, r^{i}\right)$. Comparing the calculated $\left(P^{i}, r^{i}\right)$ and the experimental data for the $50 \mu \mathrm{m}$ thick film with peel angle $180^{\circ}(P=0.51 \mathrm{~N} / \mathrm{mm}, r=0.12 \mathrm{~mm})$, one can find that the true values of both $\Gamma_{0}$ and $\hat{\sigma}$ do fall into the range $(0.02,0.2)$ and $(5,50)$, respectively. The neural network can be trained using both $\left(P^{i}, r^{i}\right)$ and $\left(\Gamma_{0}^{i}, \hat{\sigma}^{i}\right)$.

A two-layer feed-forward back propagation network with two inputs and two outputs is built in MATLAB version 7.0 [18]. There are seven nerve cells in the first layer and the transfer function is TANSIG. The second layer has two nerve cells and the transfer function is PURELIN. TRAINLM is used as the training function for the whole network. The sketch of the neural network is shown in Fig. 8. This network can simulate any function with two dependent

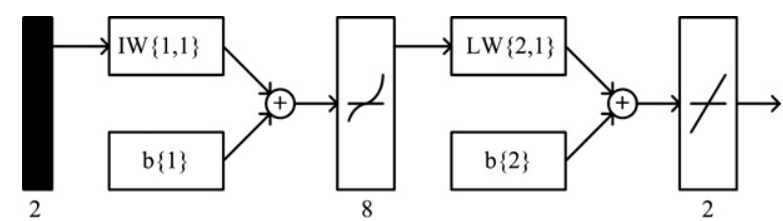

Fig. 8. Sketch of the neural network. 
and two independent variables, providing that the function is not continuous only at finite points [18].

\section{Results and discussions}

The network described in Fig. 8 is trained by using 100 values of $\left(P^{i}, r^{i}\right)$ and $\left(\Gamma_{0}^{i}, \hat{\sigma}^{i}\right)$, noting that these values are obtained based on the finite element calculations. The variations of $g_{2}$ and $f_{2}$ based on the neural network method are shown in Figs. 9 and 10, respectively. In these figures, $\Gamma$ and $\sigma$ stand for the values of $\left(\Gamma_{0}, \hat{\sigma}\right)$ to be determined, and $T$ is target value.

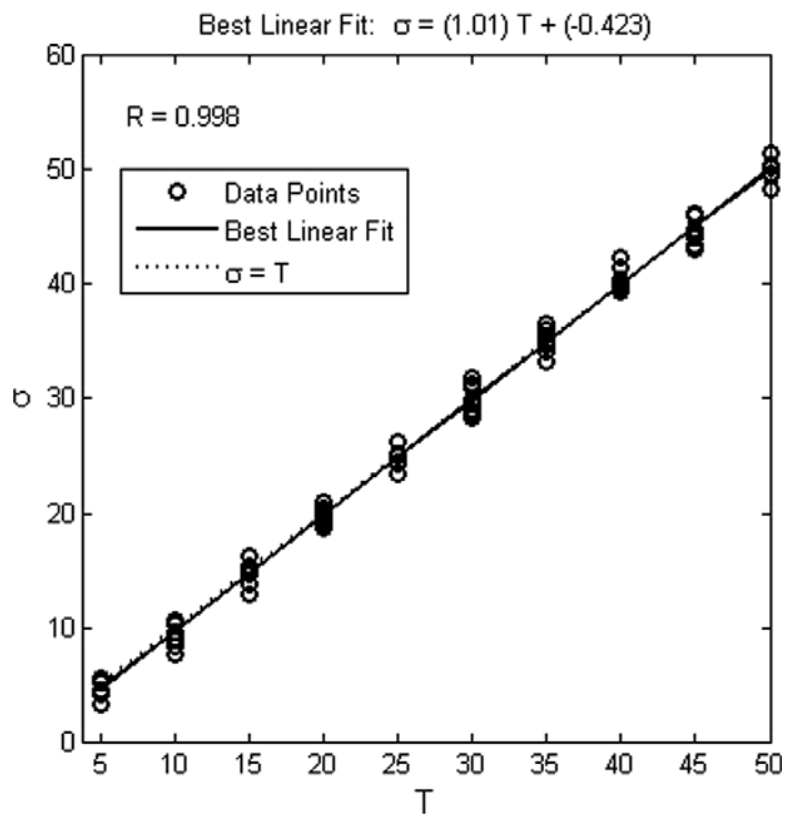

Fig. 9. The effect of simulating $g_{2} . \sigma$ is predicted values by the network with the input data $\left(P^{i}, r^{i}\right)$. T is target values and $R=0.998$ is the correlation coefficient of $\sigma$ and $T$.

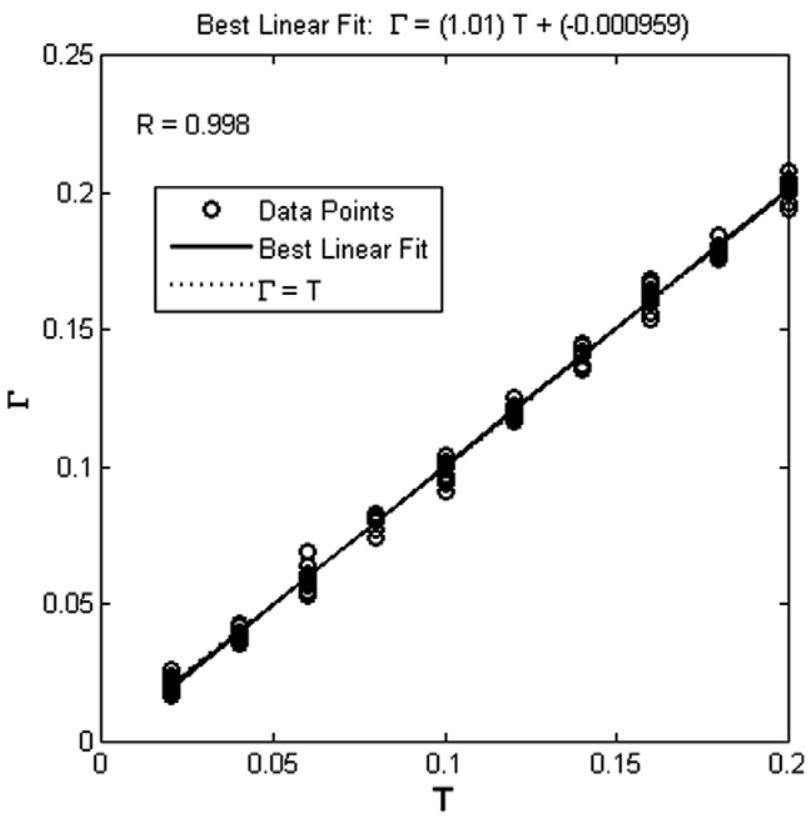

Fig. 10. The effect of simulating $f_{2}$. $\Gamma$ is predicted values by the network with the input data $\left(P^{i}, r^{i}\right) . T$ is target values and $R=0.998$ is the correlation coefficient of $\Gamma$ and $T$.
From Figs. 9 and 10, obviously, the simulated $f_{2}$ and $g_{2}$ by using the trained neural network are considerably accurate. By inputting the experimental data $(P=0.51 \mathrm{~N} / \mathrm{mm}, r=0.12 \mathrm{~mm})$ into the trained network, one can obtain $\Gamma_{0}=0.12 \mathrm{~N} / \mathrm{mm}, \hat{\sigma}=28 \mathrm{MPa}$.In order to validate the cohesive parameters obtained in above Subsection 3.2, the peel tests with other film thicknesses and peel angles are predicted using the FE model with adopting above determined cohesive parameters. Fig. 11 shows the variation of the peel force as a function of the film thickness for various peel angles. The experimental results are also shown. From Fig. 11, it can be seen that the FE result captures the trend of experimental results. It is

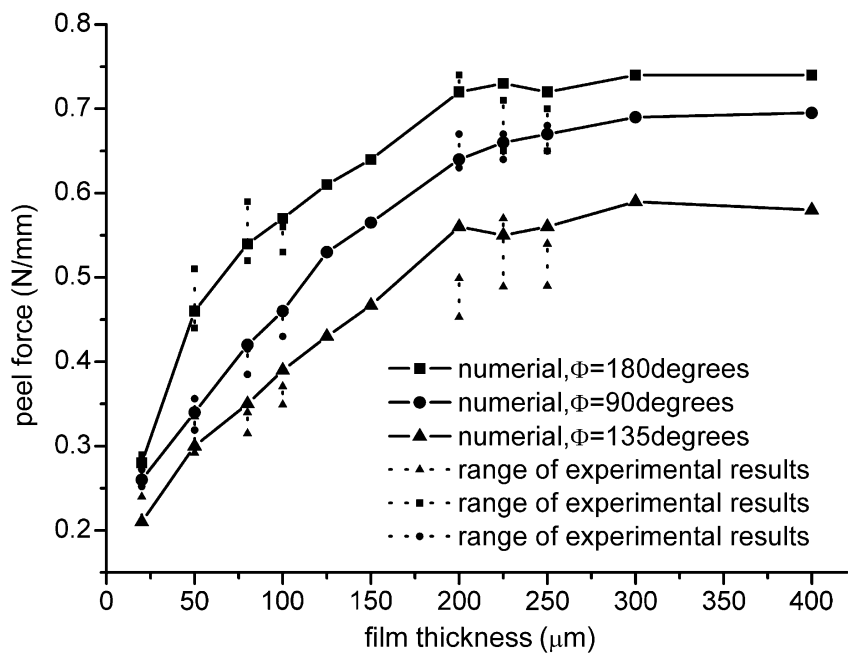

Fig. 11. The variation of the peel force as a function of the film thickness.
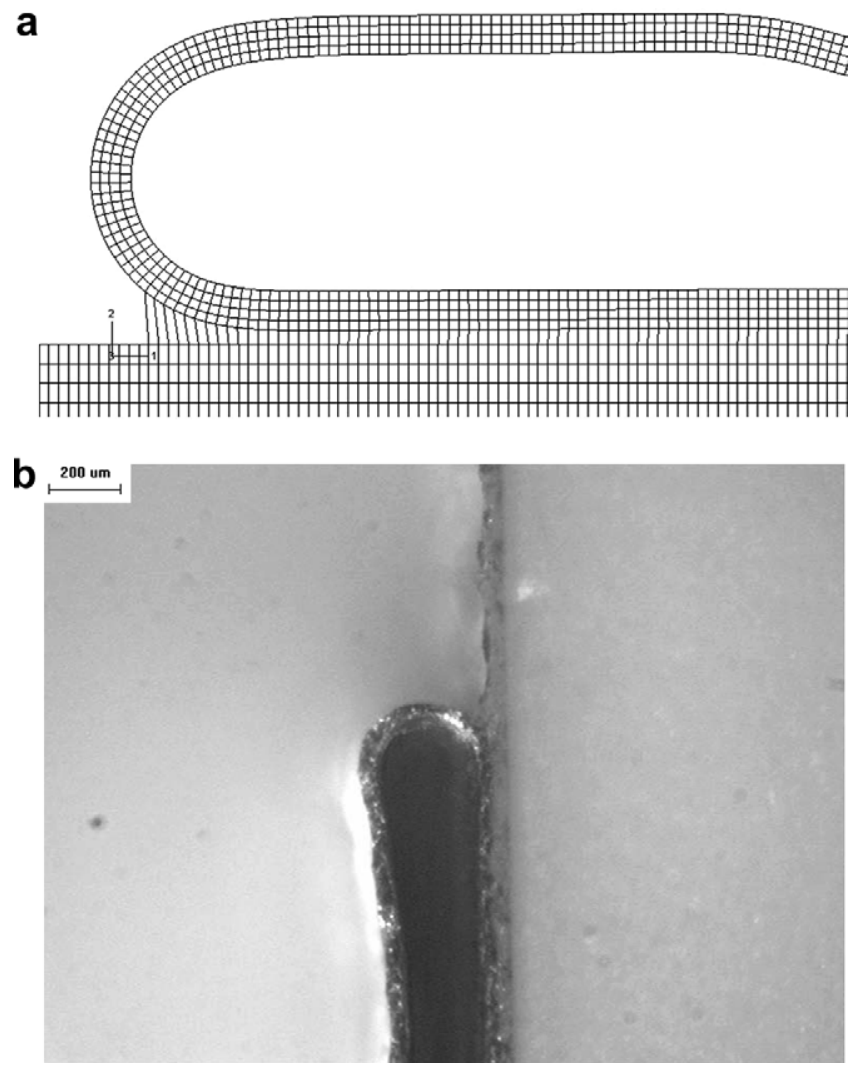

Fig. 12. Configuration of the film at the crack tip, film thickness $=50 \mu \mathrm{m}$, peel angle $=180^{\circ}$ : (a) FE simulation, and (b) experiment. 
found that once $\left(\Gamma_{0}, \hat{\sigma}\right)$ are determined for one case of film thickness and peel angle, the result can be suitable for other cases of the film thicknesses and peel angles. It seems to conclude that the fracture toughness $\Gamma_{0}$ and the separation stress $\hat{\sigma}$ can be taken as the intrinsic interfacial parameters which are independent of the film thickness and peel angle.

Fig. 12a shows the simulated configuration of the film at the crack tip. An experimental photograph is shown in Fig. 12b. From FE simulation, the bending curvature radius $r_{1}$ of the film at the crack tip is about $116 \mu \mathrm{m}$ (see Fig. 12a). The range of $r_{1}$ from experiment is $105-125 \mu \mathrm{m}$ (see Fig. 12b). The FE model captures both the steady-state peeling force and the deformation features of the film.

\section{Conclusion}

Peel test measurements for the Al film delamination along the ceramic substrate with different peel angles and different film thicknesses have been performed. The interface toughness and separation strength have been determined.

A FE model with the cohesive zone elements is used to simulate the peel test process. The FE results are used to train a neural network. The trained network is adopted to predict the interfacial cohesive energy $\Gamma_{0}$ and separation strength $\hat{\sigma}$ for the film/substrate system.

From the present research, we have noted that the FE model and the inverse analysis can effectively capture the peel test features for both the steady-state peel force and the film deformation. Both the cohesive energy $\Gamma_{0}$ and the separation strength $\hat{\sigma}$ can be taken as the intrinsic interfacial parameters which are independent of the film thickness and peel angle.

\section{Acknowledgements}

This work is supported partially by the Chinese Academy of Sciences through Grant KJCX2-YW-M04 and partially from National
Science Foundation of China through Grants Nos. 10432050, 10428207 and 10672163.

\section{References}

[1] Cotterell B, Hbaieb K, Williams JG, Hadavinia H, Tropsa V. The root rotation in double cantilever beam and peel tests. Mech Mater 2006;38:571-80.

[2] Hadavinia H, Kawashita L, Kinloch AJ, Moore DR, Williams JG. A numerical analysis of the elastic-plastic peel test. Eng Fract Mech 2006;73:2324-35.

[3] Pardoen T, Ferracin T, Landis CM, Delannay F. Constraints effects in adhesive joint fracture. J Mech Phys Solid 2005;53:1951-83.

[4] Song JY, Jin $\mathrm{Yu}$. Analysis of the T-peel strength in a $\mathrm{Cu} / \mathrm{Cr} / \mathrm{Polyimide}$ system. Acta Mater 2002;50:3985-94.

[5] Park IS, Jin Yu. An X-ray study on the mechanical effects of the peel test in a $\mathrm{Cu}$ / Cr/polyimide system. Acta Mater 1998;46:2947-53.

[6] Wei Y. Thin layer splitting along the elastic-plastic solid surface. Int J Fract 2002;113:233-52.

[7] Wei Y. Modeling nonlinear peeling of ductile thin films - critical assessment of analytical bending models using $\mathrm{FE}$ simulations. Int $\mathrm{J}$ Solid Struct 2004;41:5087-104.

[8] Yang QD, Thouless MD. Mixed-mode fracture analyses of plastically-deforming adhesive joints. Int J Fract 2001;110:175-87.

[9] Yang QD, Thouless MD, Ward SM. Numerical simulations of adhesively-bonded beams failing with extensive plastic deformation. J Mech Phys Solid 1999;47:1337-53.

[10] Yang QD, Thouless MD, Ward SM. Elastic-plastic mode-II fracture of adhesive joints. Int J Solid Struct 2001;38:3251-62.

[11] Park YB, Park IS, Jin Yu. Interfacial fracture energy measurement in the $\mathrm{Cu} / \mathrm{Cr} /$ polyimide system. Mater Sci Eng A: Struct 1999;266:261-6.

[12] Ferracin T, Landis CM, Delannay F, Pardoen T. On the determination of the cohesive zone properties of an adhesive layer from the analysis of the wedgepeel test. Int J Solid Struct 2003;40:2889-904.

[13] Dillard DA, Pocius AV. The mechanics of adhesion. 1st ed. Elsevier; 2002

[14] Wei Y, Hutchinson JW. Interface strength, work of adhesion and plasticity in the peel test. Int J Fract 1998;93:315-33.

[15] Zhao H, Wei Y. Determination of interface properties between micron-thick metal film and ceramic substrate using peel test. Int J Fract 2007:144:103-12.

[16] Wei Y, Zhao H, Shu S. Measurements and simulations of interface behavior in metal thin film delamination along ceramic substrate. In: Yilong Bai, editor. IUTAM symposium on mechanical behavior and micro-mechanics of nanostructured materials. Beijing: Springer; 2007. p. 61-70.

[17] Tvergaard V, Hutchinson JW. The influence of plasticity on mixed mode interface fracture. J Mech Phys Solid 1993;41:1119-35.

[18] MATLAB Inc., MATLAB Version 7.0 Documentation; 2004. 